\title{
Gesundheitspersonalmangel in der Schweiz beheben
}

In einem im Januar 2012 lancierten Manifest fordert eine breite Koalition, dass die Schweiz ihre Verantwortung beim globalen Mangel an Gesundheitspersonal wahrnimmt. Dazu muss sie die Ausbildung von Gesundheitsfachleuten in der Schweiz verstärken, gute Arbeitsbedingungen im Gesundheitsbereich sicherstellen und sich international stärker für die Gesundheit der Ärmsten einsetzen. Die Schweizerische Gesellschaft für Allgemeine Innere Medizin schliesst sich den Initianten des Manifests an.

\section{Jean-Michel Gaspoz ${ }^{a}$,} Lukas Zemp ${ }^{b}$

a Vorsitzender des Präsidiums der Schweizerischen Gesellschaft für Allgemeine Innere Medizin SGIM

b Generalsekretär, Geschäftsstellenleiter SGIM
Korrespondenz: SGIM

Lukas Zemp Solothurnerstrasse 68 Postfach 422 CH-4008 Basel Tel. 0612259330 Fax 0612259331 info[at]sgim.ch
Das Schweizer Gesundheitswesen ist mit einem Mangel an Fachleuten konfrontiert. Jährlich bildet es rund 5000 Personen zu wenig aus. Zudem wurden schon 201044 Prozent der Bewilligungen zur Berufsausübung an Ärzte erteilt, die im Ausland ausgebildet worden waren. Ganz abgesehen von allfälligen Sprachbarrieren dürfte dieses Potential langfristig begrenzt sein. Mit dem Gesundheitspersonalmangel ist einer der Grundpfeiler unserer Gesundheitsversorgung gefährdet. Die Schweiz steht damit nicht allein da: Der Gesundheitspersonalmangel ist ein weltweites Problem. Global gesehen stellt deshalb die Rekrutierung im Ausland auch keine ethisch vertretbare Lösung dar. Auf Initiative des Schweizer Berufsverbands der Pflegefachfrauen und Pflegefachmänner (SBK) und des Netwerks Medicus Mundi Schweiz haben 26 Organisationen, die in der Entwicklungszusammenarbeit international sowie als Berufsverbände und Gewerkschaften in der Schweiz engagiert

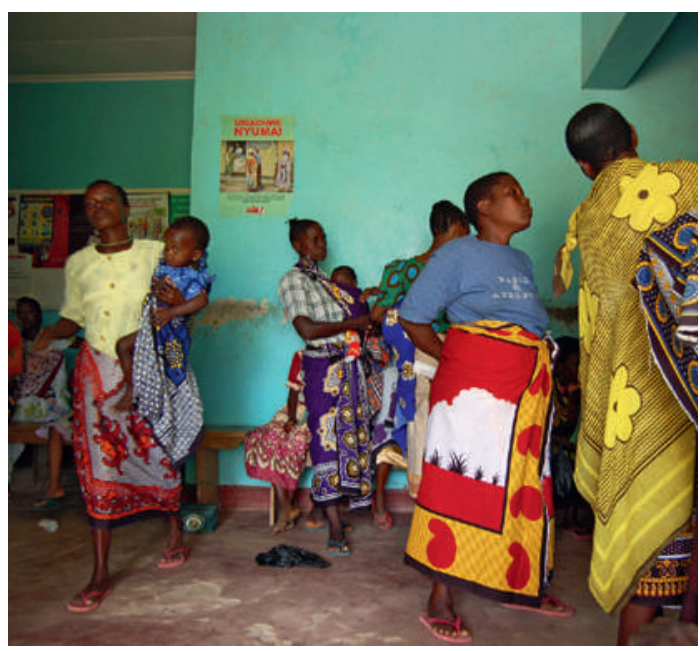

Die Schweiz kann mehr leisten, um die Gesundheitssysteme in Entwicklungsländern zu verbessern und so die Auswanderung von Personal zu verhindern. sind, im Januar 2012 ein Manifest lanciert. Nach Ansicht dieser Koalition muss die Schweiz mehr tun, um dem dramatischen Mangel an Gesundheitspersonal zu begegnen und dem WHO-Kodex auch in der Schweiz zum Durchbruch zu verhelfen. Die Schweizerische Gesellschaft für Allgemeine Innere Medizin (SGIM) hat sich den Initianten des Manifests im Herbst 2012 angeschlossen, weil deren Anliegen mit den Anliegen und dem Engagement der SGIM übereinstimmen.

\section{Langfristiges Engagement statt kurzfristiges Denken}

Im Manifest kritisieren die beteiligten Organisationen, dass die Schweiz nur schon aus demografischen Gründen mehr Personal bräuchte. Doch die Politik hat es unterlassen, durch Steuerungsmassnahmen die Situation zu entschärfen, indem etwa genügend Studienplätze für Ärztinnen und Ärzte und anderes medizinisches Fachpersonal geschaffen werden.

Das Manifest sieht auf verschiedenen Ebenen Handlungsbedarf: Die Schweiz muss bessere Steuerungs- und Planungsinstrumente entwickeln, mehr in die Ausbildung investieren und qualitativ gute Arbeitsstellen sichern, damit mehr Fachpersonen im Gesundheitssektor bleiben. Die Kompetenzen der hier lebenden Migrantinnen und Migranten müssen gestärkt und fürs Gesundheitswesen besser genutzt werden. Um der globalen Dimension des Problems gerecht zu werden, muss das Gesundheitspersonal in Entwicklungsländern bessere Perspektiven vor Ort erhalten, damit es nicht auswandert. Die Schweiz kann hier wesentlich mehr leisten, indem sie sich verstärkt für die Verbesserung der Gesundheitssysteme in Entwicklungsländern engagiert.

Mehr Informationen über das Manifest sind zu finden unter www.sgim.ch/de/publikationendokumente/stellungnahmen oder www.gesundheitspersonalmangel.wordpress.com 